\title{
Are patients with alcohol-related fatty liver at increased risk of coronary heart disease?
}

\author{
Dong Hyun Sinn and Yong-Han Paik \\ Department of Internal Medicine, Samsung Medical Center, Sungkyunkwan University School of Medicine, Seoul, Korea
}

Keywords: Fatty liver; Coronary heart disease; Alcohol

\section{See Article on Page 154}

Hepatic steatosis is defined when the deposition of triglyceride (TG) as lipid droplets in the cytoplasm of hepatocytes in more than $5 \%$ of hepatocytes. ${ }^{1}$ TG is a preferred storage nutrient as it provide greater caloric density $(9 \mathrm{kcal} / \mathrm{g}$ ) than do carbohydrates $(4.5 \mathrm{kcal} / \mathrm{g})$ or protein $(4 \mathrm{kcal} / \mathrm{g})$, and is insoluble in water, so they can accumulate to high levels with no adverse osmotic or colloidal effects on cells. ${ }^{2}$ TG is usually stockpiled in adipocytes, however, it accumulates in other cell types under certain circumstances. ${ }^{2}$

A common cause for hepatic steatosis is use of significant alcohol consumption. Fatty liver develops in about $90 \%$ of individuals who drink significant amount of alcohol. ${ }^{3}$ There are some other causes for hepatic fat accumulation, such as medication, inborn errors of metabolism (e.g., cholesterol ester storage disease), Reye's syndrome, Wilson's disease, lipodystrophy, abetalipoproteinemia, etc. ${ }^{4}$ Nonalcoholic fatty liver disease (NAFLD) is also common cause of hepatic steatosis, and is defined when there are no causes for secondary hepatic fat accumulation, such as significant alcohol consumption, use of steatogenic medication or hereditary disorders. ${ }^{4}$

In humans, fatty liver can have severe clinical consequences. If underlying causes for hepatic steatosis are not corrected (e.g., abstinence in case of alcoholic fatty liver, weight reduction in case of NAFLD), progression to hepatic fibrosis and liver cirrhosis may occur. ${ }^{3,4}$ Furthermore, fatty liver is not only limited to causing hepatic problems. It is well accepted that patients with NAFLD are at increased risk of complication related to metabolic disease, in particular, cardiovascular disease..$^{5-7}$

In patients with NAFLD, insulin resistance is commonly found, and is considered as the common pathophysiological mechanism that explains metabolic derangement of the body, observed in NAFLD. ${ }^{8}$ However, whether NAFLD is a cause or consequence of insulin resistance remains unclear. If TG accumulation in hepatocytes causes insulin resistance in human, fatty liver itself, independent of causes for hepatic steatosis, will be associated with metabolic disease. So far, association between fatty liver and cardiovascular disease is extensively studied in patients with NAFLD, ${ }^{7,9}$ but not for fatty liver that is related to significant alcohol consumption.

In this issue of the Clinical and Molecular Hepatology, Kim et al, assessed whether alcoholic fatty liver is associated with coronary heart disease risk. ${ }^{10}$ They analyzed the association between hepatic steatosis and coronary heart disease risk in 10,710 health check-up participants. Hepatic steatosis was diagnosed with ultrasonography, and individuals were divided into alcoholic fatty liver

\section{Abbreviations:}

NAFLD, nonalcoholic fatty liver disease; TG, triglyceride

\author{
Corresponding author : Yong-Han Paik \\ Department of Internal Medicine, Samsung Medical Center, \\ Sungkyunkwan University School of Medicine, 81 Irwon-ro, Gangnam-gu, \\ Seoul 135-710, Korea \\ Tel. +82-2-3410-3409, Fax. +82-2-3410-6983 \\ E-mail; yh.paik@skku.edu
}


and nonalcoholic fatty liver, according to the amount of alcohol consumption. Coronary heart disease risk was estimated using Framingham Risk Score. They found that alcoholic fatty liver was also associated with increased estimated coronary heart disease risk as in NAFLD (Framingham Risk Score: 7.32 $\pm 0.07,6.72 \pm 0.12$ and $5.50 \pm 0.04$ for NAFLD, alcoholic fatty liver and control, respectively). The prevalence of the estimated total coronary heart disease risk over $20 \%$ was significantly higher in individuals with fatty liver compared to control (4.7\% vs. $2.0 \%, P<0.001)$, and was comparable between NAFLD and alcoholic fatty liver $(5.2 \%$ vs. $3.4 \%, P=0.05)$.

Does this data mean that we need to screen cardiovascular disease in alcohol-related fatty liver as in NAFLD patients? Apart from some of limitations authors have discussed, ${ }^{10}$ some more points need to be considered. Most importantly, the association between alcoholic fatty liver and cardiovascular disease mortality needs to be demonstrated. Contrast to NAFLD where increased cardiovascular disease mortality has been demonstrated, ${ }^{7}$ whether patients with alcohol-related fatty liver are at increased risk of death from cardiovascular disease remains unknown. Heavy alcohol drinking is associated with metabolic syndrome, ${ }^{11}$ and obesity, ${ }^{12,13}$ which are strong risk factors for cardiovascular disease. Heavy alcohol drinking also increases the progression of atherosclerosis, ${ }^{14}$ and this phenomenon appears to be due to the metabolism of ethanol, that leads to the formation of acetaldehyde, which is oxidized to acetate, leading to the generation of reactive oxygen species and a toxic effect of alcohol on the formation of the atherosclerosis plaque. ${ }^{14}$ Increased cardiovascular risk observed in alcohol-related fatty liver, may be from heavy alcohol use, apart from hepatic steatosis. To make things more complex, when used in modest amount, alcohol shows favorable pleiotropic effects on lipids, adhesion molecules, platelet activation and oxidative stress, ${ }^{14}$ and people who consumed one alcoholic drink daily had a decrease of $30-40 \%$ in cardiovascular mortality compared with nondrinkers. ${ }^{15}$ Therefore, more data is needed to understand complex relationship between hepatic steatosis, amount of alcohol and cardiovascular disease. Nevertheless, the study from Kim et al, calls our attention for cardiovascular health in individuals with alcohol-related fatty liver. Appropriate assessment of cardiovascular system is warranted for these patients.

\section{Conflicts of Interest}

The authors have no conflicts to disclose.

\section{REFERENCES}

1. Szczepaniak LS, Nurenberg $P$, Leonard D, Browning JD, Reingold $J S$, Grundy $S$, et al. Magnetic resonance spectroscopy to measure hepatic triglyceride content: prevalence of hepatic steatosis in the general population. Am J Physiol Endocrinol Metab 2005;288:E462 E468.

2. Cohen JC, Horton JD, Hobbs HH. Human fatty liver disease: old questions and new insights. Science 2011;332:1519-1523.

3. O'Shea RS, Dasarathy S, McCullough AJ. Alcoholic liver disease. Am J Gastroenterol 2010;105:14-32; quiz 33.

4. Chalasani N, Younossi Z, Lavine JE, Diehl AM, Brunt EM, Cusi K, et al. The diagnosis and management of non-alcoholic fatty liver disease: practice Guideline by the American Association for the Study of Liver Diseases, American College of Gastroenterology, and the American Gastroenterological Association. Hepatology 2012;55:2005-2023.

5. Lazo M, Hernaez R, Bonekamp S, Kamel IR, Brancati FL, Guallar $E$, et al. Non-alcoholic fatty liver disease and mortality among US adults: prospective cohort study. BMJ 2011;343:d6891.

6. Calori G, Lattuada G, Ragogna F, Garancini MP, Crosignani P, Villa $M$, et al. Fatty liver index and mortality: the Cremona study in the 15th year of follow-up. Hepatology 2011;54:145-152.

7. Del Ben M, Baratta F, Polimeni L, Angelico F. Non-alcoholic fatty liver disease and cardiovascular disease: epidemiological, clinical and pathophysiological evidences. Intern Emerg Med 2012;7(Suppl 3):S291-\$296.

8. Bugianesi E, McCullough AJ, Marchesini G. Insulin resistance: a metabolic pathway to chronic liver disease. Hepatology 2005;42:987-1000.

9. Sinn DH, Gwak GY, Cho J, Son HJ, Paik YH, Choi MS, et al. Modest alcohol consumption and carotid plaques or carotid artery stenosis in men with non-alcoholic fatty liver disease. Atherosclerosis 2014;234:270-275.

10. Kim HJ, Kim JH, Choe WH, Kown SY, Lee CH. Alcoholic fatty liver disease elevates estimated coronary heart disease risk comparable to nonalcoholic fatty liver disease in the Korean population: A cross sectional study. Clin Mol Hepatol 2014;20:154-161.

11. Sun K, Ren M, Liu D, Wang C, Yang C, Yan L. Alcohol consumption and risk of metabolic syndrome: A meta-analysis of prospective studies. Clin Nutr 2013 Oct 14. pii: S0261-5614(13)00262-8. doi: 10.1016/j.clnu.2013.10.003. [Epub ahead of print]

12. Shelton NJ, Knott CS. Association between alcohol calorie intake and overweight and obesity in English adults. Am J Public Health 2014;104:629-631.

13. Croezen S, Visscher TL, Ter Bogt NC, Veling ML, Haveman-Nies A. Skipping breakfast, alcohol consumption and physical inactivity as risk factors for overweight and obesity in adolescents: results of the 
Dong Hyun Sinn, et al. Are patients with alcohol-related fatty liver at increased risk of coronary heart disease?

E-MOVO project. Eur J Clin Nutr 2009;63:405-412.

14. Carnevale R, Nocella C. Alcohol and cardiovascular disease: still unresolved underlying mechanisms. Vascul Pharmacol 2012;57:6971.
15. Thun MJ, Peto R, Lopez AD, Monaco JH, Henley SJ, Heath CW Jr, et al. Alcohol consumption and mortality among middle-aged and elderly U.S. adults. N Engl J Med 1997;337:1705-1714. 